\title{
POLYGENIC HYPERLIPIDEMIA INCREASES CORONARY ARTERY DISEASE RISK IN THE UK BIOBANK \& FINNGEN
}

Pietari Ripatti, Joel T Rämö, Sanni Söderlund, Ida Surakka, Aki S Havulinna, Elisabeth Widén, Priit Palta, Nelson B Freimer, Veikko Salomaa, Matti Pirinen, Aarno Palotie, Marja-Riitta Taskinen, and Samuli Ripatti

\section{Polygenic risk scores (PRS) for LDL-C and TG}

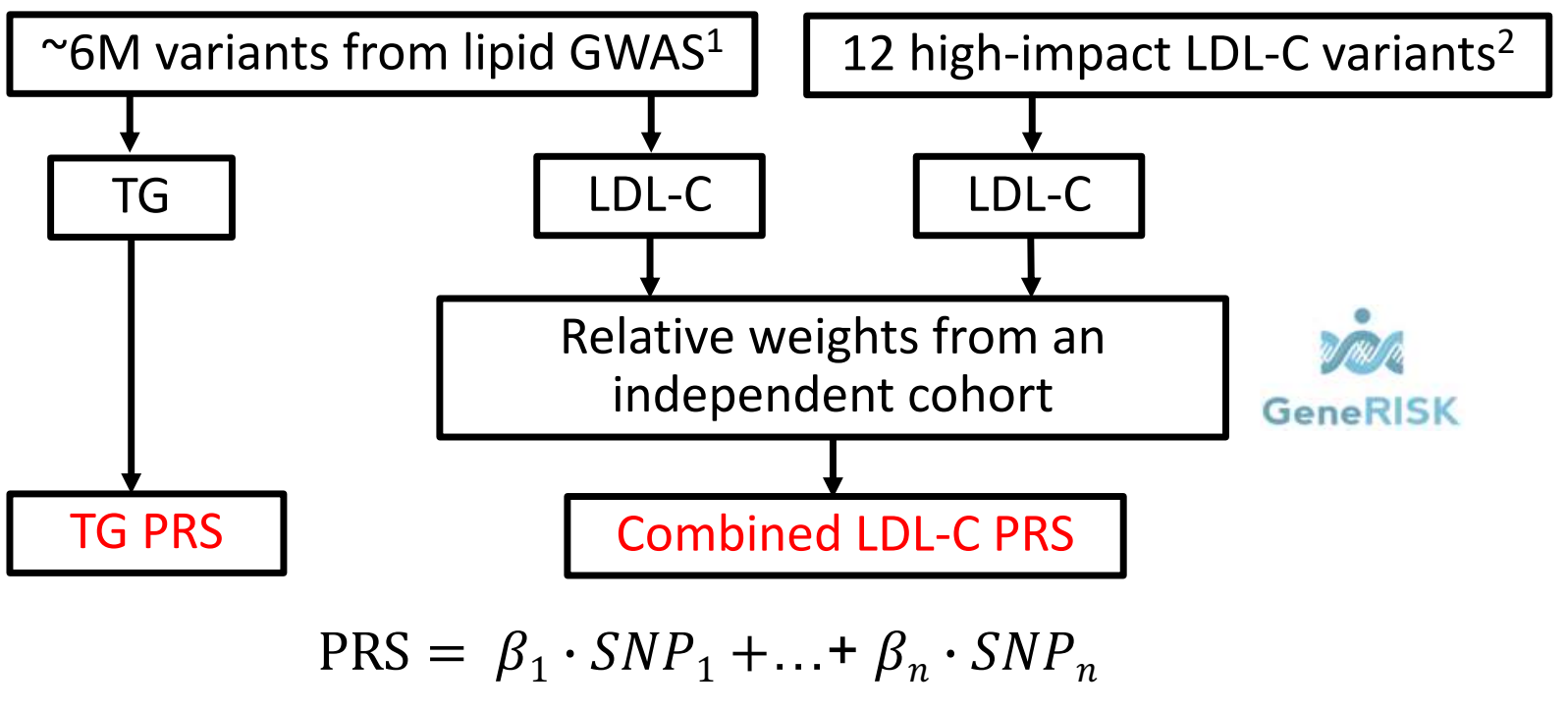

Three cohorts with 440,171 individuals
FINRISKI

The National

FINRISK Study

- Finnish

population

cohort

- $n=27039$

with lipid

measures

\section{biobank"}

FinnGen Project
- $n=96499$
with
longitudinal
registry data
- 7123 CAD cases

UK Biobank

- $n=343672$

with registry data

- 16370 CAD cases
$C A D=M A C E=M I$ or revascularization (registry-based or self-reported)

LDL-C PRS associates with a $1.3 \mathrm{mmol} / \mathrm{I}$ difference in LDL-C and $23-63 \%$ increased CAD risk

Between the highest and lowest 5\%, LDL-C differed by $1.3 \mathrm{mmol} / \mathrm{l}$ and CAD risk increased by $23-63 \%$.

The effect was less pronounced than reported for monogenic $\mathrm{FH}(1.8 \mathrm{mmol} / \mathrm{l}$ elevation in LDL-C and $160 \%$ elevation in CAD risk). ${ }^{3}$
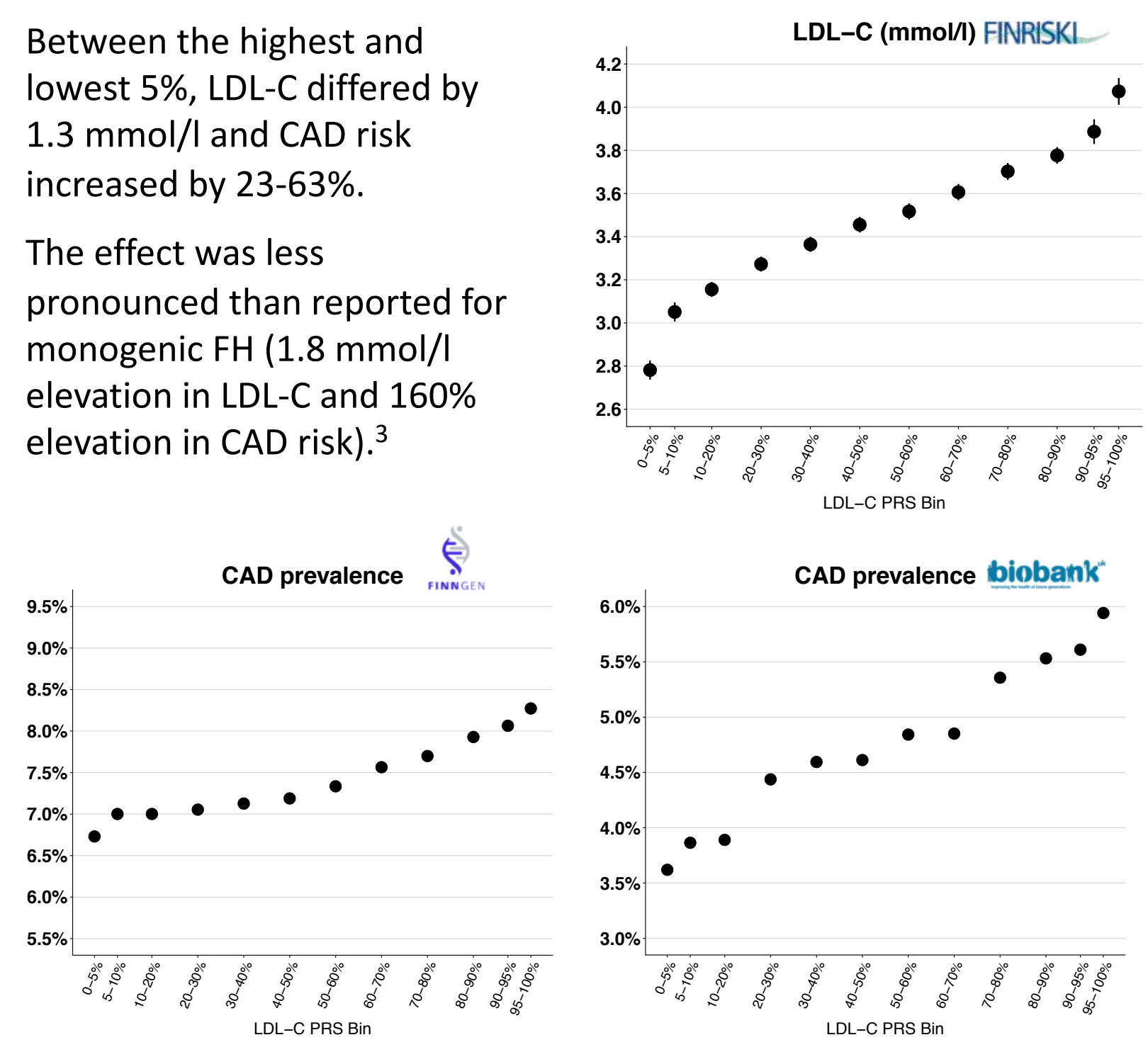

TG PRS associates with a $1.0 \mathrm{mmol} / \mathrm{l}$ difference in TGs and 51-57\% increased CAD risk

Between the highest and lowest $5 \%$, TG differed by $1.0 \mathrm{mmol} / \mathrm{l}$ and CAD risk increased by 51 $57 \%$.

The effect was in line with lossof-function mutations in $A P O C 3$ or ANGPTL4 (0.7-0.8 mmol/l reductions in TGs and $36-53 \%$ reductions in CAD risk). ${ }^{4-5}$
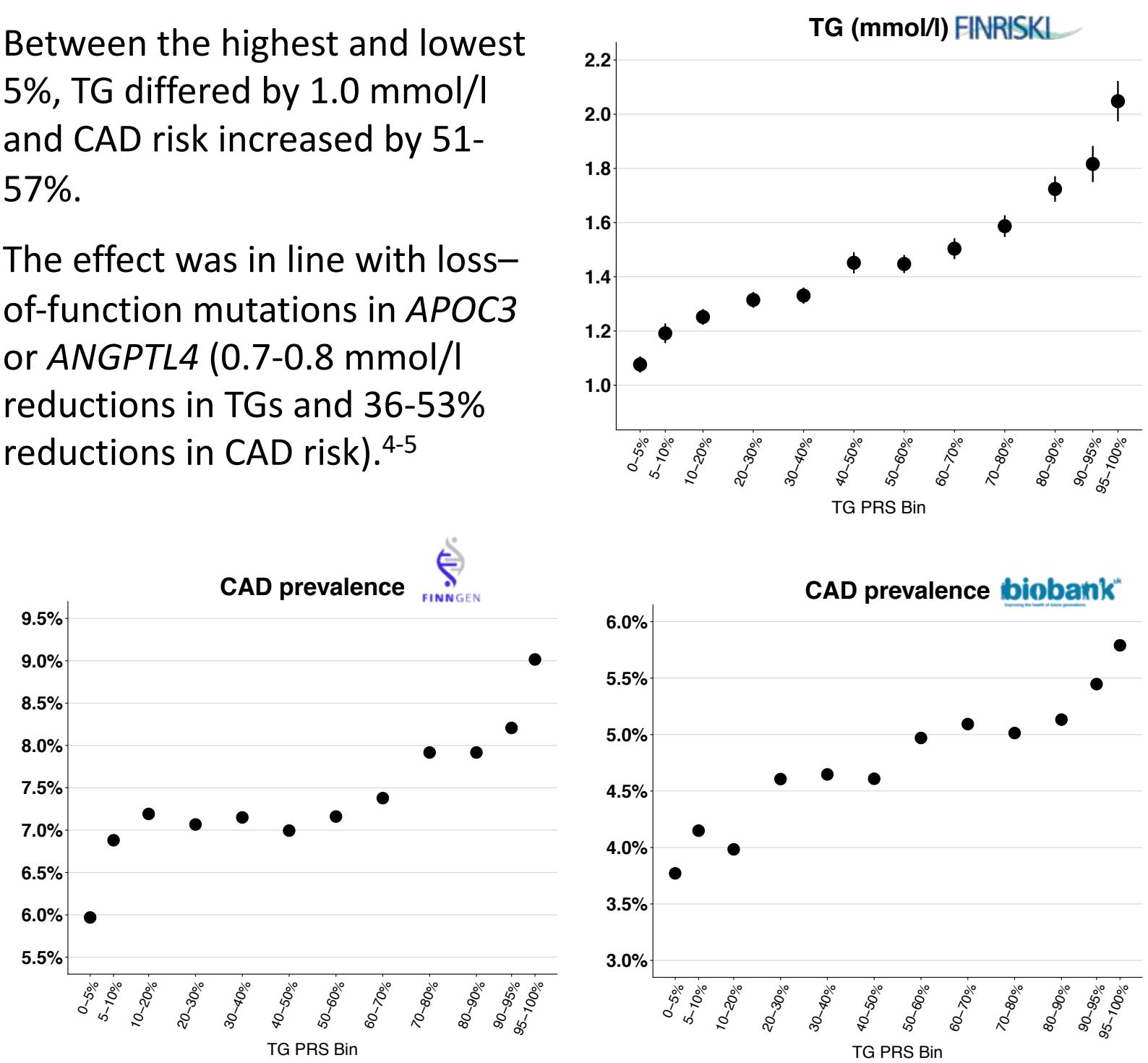

\section{PRSs for LDL-C \& TGs are mostly independent of a CAD PRS}

We constructed a PRS directly for CAD. ${ }^{6}$

OR for CAD between the highest and lowest $5 \%$ of the lipid PRS percentiles remained high after adjusting for the CAD PRS.

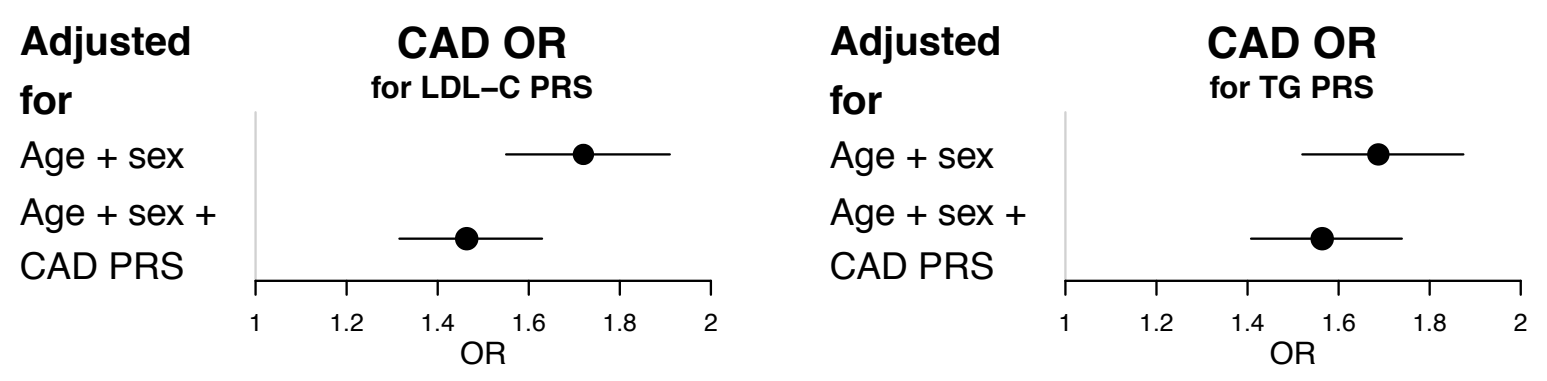

\section{CONCLUSIONS}

1. High polygenic burden significantly elevates LDL-C and TG levels

2. Polygenic hypercholesterolemia associates with less pronounced LDL-C \& CAD risk elevation compared with monogenic $\mathrm{FH}$

3. Polygenic hyperlipidemia increases CAD risk mostly independently of a PRS for CAD

\section{References:}

1. Surakka et al. Nat Genet. 2015 Jun;47(6):589-97

2. Talmud et al. Lancet. 2013 Apr 13;381(9874):1293-301.

3. Abul-Husn, NS et al. Science. 2016:354(6319).

4. Jørgensen, AB et al. N Engl J Med. 2014;371:32-41.

5. 2MI Genetics and CARDIoGRAM Exome Consortia Investigators. N Engl J Med. 2016;374:1134-1144.

6. Nikpay M et al. Nat Genet. 2015 Oct;47(10):1121-1130. 